\title{
The use of pulse wave velocity in predicting pre-eclampsia in high-risk women
}

\author{
Irene Katsipi ${ }^{1}$, Kostas Stylianou ${ }^{1}$, Ioannis Petrakis ${ }^{1}$, Andrew Passam ${ }^{1}$, Eleftheria Vardaki ${ }^{1}$, \\ Fragkiskos Parthenakis ${ }^{2}$, Antonios Makrygiannakis ${ }^{3}$, Eugene Daphnis ${ }^{1}$ and John Kyriazis ${ }^{4}$
}

In this study, we evaluated the diagnostic utility of pulse wave velocity (PWV) alone or in combination with other diagnostic markers in predicting pre-eclampsia (PE) in high-risk women. Pregnant women at high risk for PE were recruited between 22 and 26 weeks of gestation and were assessed for (a) PWV, (b) serum levels of the placental soluble fms-like tyrosine kinase 1 (sFlt-1) protein and uric acid and (c) 24-h urinary protein and calcium excretion. Sensitivities and specificities were derived from receiver operating characteristic curves. Of 118 women recruited, 11 and 10 women developed early-onset PE ( $<34$ weeks) and late-onset PE ( $\geq 34$ weeks), respectively. Of the five diagnostic markers tested, PWV showed the highest detection rate for all cases $(21)$ of PE (81\%) and for early-onset PE (82\%) at a fixed $10 \%$ false-positive rate (FPR), and when combined with sFIt-1, these figures increased to $90 \%$ and $92 \%$, respectively. Despite the reduced ability of PWV to predict late-onset PE (detection rate $20 \%$ ), the combination of PWV with sFIt-1 achieved a detection rate of $50 \%$ at a fixed $10 \%$ FPR. A suggested cutoff value of $9 \mathrm{~m} / \mathrm{s}$ for PWV resulted in optimal sensitivity $(91 \%)$ and specificity $(86 \%)$ for predicting early-onset PE. This study is the first to show that PWV may be a potentially promising predictor of early-onset PE in women at high risk for PE. The combination of PWV with sFIt-1 may further improve the screening efficacy for predicting PE. Hypertension Research (2014) 37, 733-740; doi:10.1038/hr.2014.62; published online 13 March 2014

Keywords: predictive value of tests; pre-eclampsia; pregnancy; pulse wave velocity

\section{INTRODUCTION}

Pre-eclampsia (PE), a syndrome of pregnant women, complicates $\sim 5-8 \%$ of all pregnancies and is a major cause of maternal morbidity and mortality worldwide. ${ }^{1,2}$ It is defined as the onset of hypertension and proteinuria after the 20th week of gestation in a previously normotensive and non-proteinuric pregnant woman. ${ }^{1}$ The pathophysiology of PE likely involves both fetal/placental and maternal factors. When occurring early in pregnancy, placental underperfusion upregulates the production of placental anti-angiogenic factors - such as soluble fms-like tyrosine kinase 1 (sFlt-1), which is the truncated form of the full-length vascular endothelial growth factor receptor type-1 - that alter maternal systemic endothelial function and cause hypertension, proteinuria and the other systemic manifestations of the disease. ${ }^{3-6}$ There is substantial evidence ${ }^{6,7}$ suggesting that the excessive amount of sFlt-1 secreted by the ischemic placenta leads to a generalized antiangiogenic state (otherwise known as 'a generalized endothelial dysfunction') by impairing the signaling of the angiogenic factors vascular endothelial growth factor and placental growth factor.

Early prediction of the disease may facilitate initiation of timely management to avert mortality and morbidity of the mother and baby. Currently, multiple risk factors for the development of PE have been described, ${ }^{8}$ and a variety of markers have been proposed for its early diagnosis. ${ }^{9}$ However, systematic reviews of relevant studies ${ }^{10,11}$ concluded that none of the evaluated biochemical and biophysical markers have been proven to be accurate predictive tool for PE. Thus, combinations of biochemical and biophysical markers have been used extensively to improve early prediction of PE. ${ }^{12}$

To achieve higher predictive power, combinations of markers, which generally lead to an increase in sensitivity and/or specificity compared with single markers, are of interest, but new potential predictors are also of importance. In this regard, there is emerging evidence that PE is associated with increased arterial stiffness (AS), which is itself an important predictor of outcome. ${ }^{13}$ A recent metaanalysis of 23 relevant studies showed a significant increase in all AS indices measured in pre-eclamptic compared with normotensive pregnant women. ${ }^{14}$ Aortic stiffness reportedly varies throughout normal pregnancy, reaching its nadir in the second trimester and rising again in the third, ${ }^{15,16}$ but in pre-eclamptic women it continues to increase throughout pregnancy. ${ }^{15}$ Because the changes in AS throughout pre-eclamptic pregnancy differ from those of normal pregnancy, this temporal relationship could be used to predict pregnancy outcomes.

\footnotetext{
${ }^{1}$ Department of Nephrology, University Hospital of Heraklion, Heraklion, Crete, Greece; ${ }^{2}$ Department of Cardiology, University Hospital of Heraklion, Heraklion, Crete, Greece; ${ }^{3}$ Department of Obstetrics and Gynecology, University Hospital of Heraklion, Crete, Greece and ${ }^{4}$ Department of Nephrology, General Hospital of Chios, Chios, Greece Correspondence: Dr J Kyriazis, General Hospital of Chios, Dialysis Unit, Chios 82100, Greece.

E-mail: jks@otenet.gr

Received 12 August 2013; revised 11 November 2013; accepted 16 December 2013; published online 13 March 2014
} 
AS may be measured using a variety of different techniques, described in detail elsewhere. ${ }^{17}$ Carotid-femoral pulse wave velocity (PWV), the most widely validated and universally accepted measure of AS, is considered the 'gold-standard' measurement of AS. ${ }^{18} \mathrm{PWV}$ has not been adequately examined during pregnancy, and the potential utility of PWV as a predictor of PE has not yet been determined. The aim of this study was to (a) evaluate PWV as a marker for the early prediction of $\mathrm{PE}$, (b) compare its predictive value with that of sFlt-1 and other traditional diagnostic markers, such as serum uric acid and 24-h urine protein and calcium excretion and (c) examine whether PWV combined with other biomarkers could improve prediction of the disease.

\section{METHODS}

\section{Study population}

One hundred and eighteen pregnant women aged between 15 and 41 years were recruited consecutively from the patients presenting for prenatal care at the high-risk obstetrics clinic of the University Hospital of Heraklion. Inclusion criteria were pregnancy between 22 and 26 weeks of gestation at enrollment and at least one of the following risk factors for PE: nulliparity, pregestational diabetes mellitus, obesity, chronic hypertension, pregestational proteinuria, young ( $<18$ years) and old ( $>35$ years) maternal age, prior or family history of PE, connective tissue disease, thrombophilia, fetal growth restriction or fetal demise in a previous pregnancy.

Diagnosis of pregestational diabetes mellitus required an abnormal glucose tolerance test or therapy with oral hypoglycemic/insulin prior to conception. Obesity was defined as a body mass index (BMI) of $30 \mathrm{~kg} \mathrm{~m}^{-2}$ or greater based on self-reported pre-pregnancy weight and height. A diagnosis of chronic hypertension required the use of antihypertensive agents or the presence of blood pressure of $140 / 90 \mathrm{~mm} \mathrm{Hg}$ or greater on at least two occasions at least $4 \mathrm{~h}$ apart, prior to 20 weeks of gestation. Pregestational proteinuria was defined as proteinuria of $300 \mathrm{mg}$ or greater in a 24 -h urine collection prior to 20 weeks of gestation. A diagnosis of systemic connective tissue disease and thrombophilia was based on a clinical diagnosis documented in the medical records. The presence of PE, fetal growth restriction or fetal demise in a previous pregnancy was confirmed in patient interviews or maternal chart reviews. Finally, clinical information collected at the time of study entry for each patient also included a family history of PE and smoking habits. The study protocol was approved by the local ethics committee, and informed consent was obtained from all enrolled participants.

\section{Diagnosis of PE}

The presence of $\mathrm{PE}$ and the gestational age at which it developed were determined by reviewing the medical records of each subject after the completion of pregnancy. PE was diagnosed based on the definition of the National High Blood Pressure Education Program Working Group on High Pressure in Pregnancy. ${ }^{19}$ In women without baseline hypertension or proteinuria, PE was defined as the new onset of hypertension and proteinuria after 20 weeks of gestation. Hypertension was defined as either a systolic blood pressure (SBP) of $140 \mathrm{~mm} \mathrm{Hg}$ or greater or a diastolic blood pressure of $90 \mathrm{mmHg}$ or greater on two occasions at least $4 \mathrm{~h}$ apart. Proteinuria was defined as the excretion of $300 \mathrm{mg}$ or more protein in a 24-h urine collection with no evidence of urinary tract infection. In women with chronic hypertension, the diagnosis of PE required new-onset proteinuria after 20 weeks of gestation. In women with pregestational proteinuria, the diagnosis of $\mathrm{PE}$ required new-onset hypertension and worsening proteinuria (doubling of 24-h urine protein) after 20 weeks of gestation. The onset of PE was defined as the time of the first elevated blood pressure or urinary protein measurement.

\section{Procedures}

All pregnant women were assessed, between 22 and 26 weeks of gestation, for (a) PWV, (b) serum levels of sFlt-1 and uric acid and (c) 24-h urinary protein and calcium excretion. In subjects who developed PE, all examinations were performed prior to PE onset.
PWV was recorded in the supine position using a Pulse Trace 6000 system (MicroMedical Ltd., Kent, UK) as described previously. ${ }^{20}$ All measurements were calculated in duplicate, and mean values were used in subsequent analyses. Blood pressure was measured using a mercury sphygmomanometer prior to PWV measurements. Three measurements determined at 2-min intervals were averaged. Each subject was examined under standardized conditions after a 5-min rest in the left lateral position to avoid vena cava compression by the uterus.

In this study, we additionally assessed the short-term reproducibility of PWV measurements in 20 pregnant women at 16-24 weeks of gestation by obtaining two measurements at 2 days apart under identical conditions. The reproducibility was assessed using Bland-Altman agreement analysis (Figure 1), which showed acceptable consistency between the repeated PWV measures. The mean difference between the two PWV measurements was $-0.035 \mathrm{~m} / \mathrm{s}$, and the $95 \%$ limits of agreement were $-0.90-0.83 \mathrm{~m} / \mathrm{s}$. In addition, there was an excellent correlation between the two PWV measurements $(r=0.930 ; P<0.001)$, and the variation coefficient of the repeated PWV measures was $4.06 \pm 2.33 \%$.

Blood samples were centrifuged within a maximum of $30 \mathrm{~min}$ after collection. Serum uric acid levels were then immediately measured using a conventional autoanalyzer, and the remaining serum was stored at $-80^{\circ} \mathrm{C}$ until assayed. Enzyme-linked immunosorbent assays for human sFlt-1 were performed in duplicate using commercial kits (R\&D Systems, Minneapolis, $\mathrm{MN}$, USA) as previously described ${ }^{7}$ by personnel who were unaware of the outcome of the pregnancy. Interassay and intraassay coefficients of variation were 7.4 and $3.2 \%$, respectively, and the minimal detectable concentration was $5 \mathrm{pg} \mathrm{ml}^{-1}$.

All women were asked to collect a 24 -h urine sample. Urinary protein and calcium were measured and expressed as milligrams per $24 \mathrm{~h}$.

\section{Statistical analyses}

The data were analyzed using SPSS-20.0 software (SPSS Inc., Chicago, IL USA). The results are expressed as the mean \pm s.d. for normally distributed variables and as the median (interquartile range) for non-normally distributed variables. Normally distributed variables were compared using Student's $t$-tests and non-normally distributed variables using Mann-Whitney $U$-tests. The $X^{2}$-test was used to compare categorical variables among groups. Univariate regression analysis was used to test the associations of variables correlating with PWV and sFlt-1. Non-normally distributed variables were log-transformed for regression analyses. Receiver operating characteristic (ROC) curve analysis was performed to examine the diagnostic performance of each factor in identifying patients destined to develop PE. The cutoff value for each factor

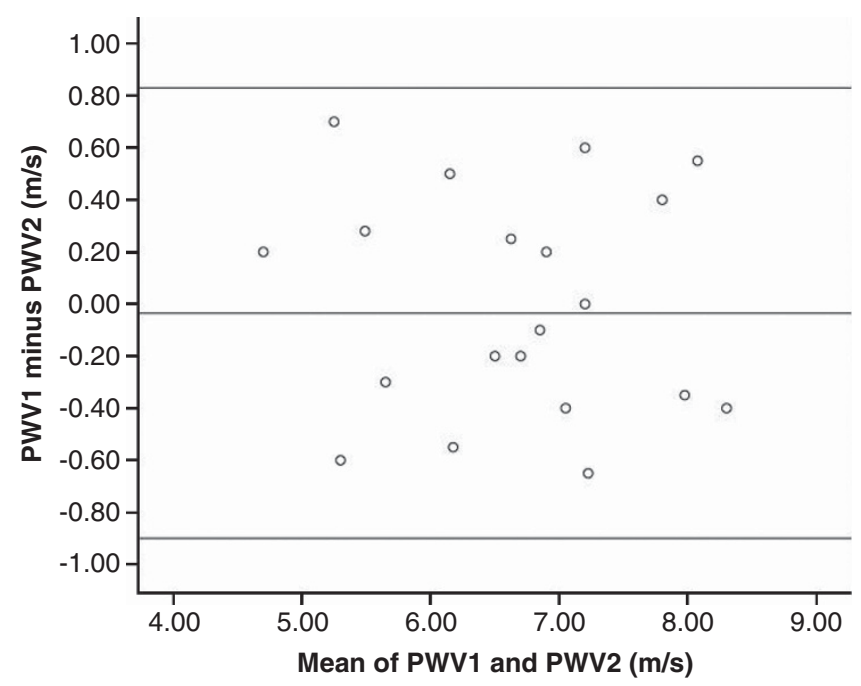

Figure 1 Bland-Altman plot showing the difference between the two measurements vs. the mean of the two measurements of PWV. 
Table 1 Demographic, clinical, hemodynamic and biochemical characteristics of study subjects at enrollment

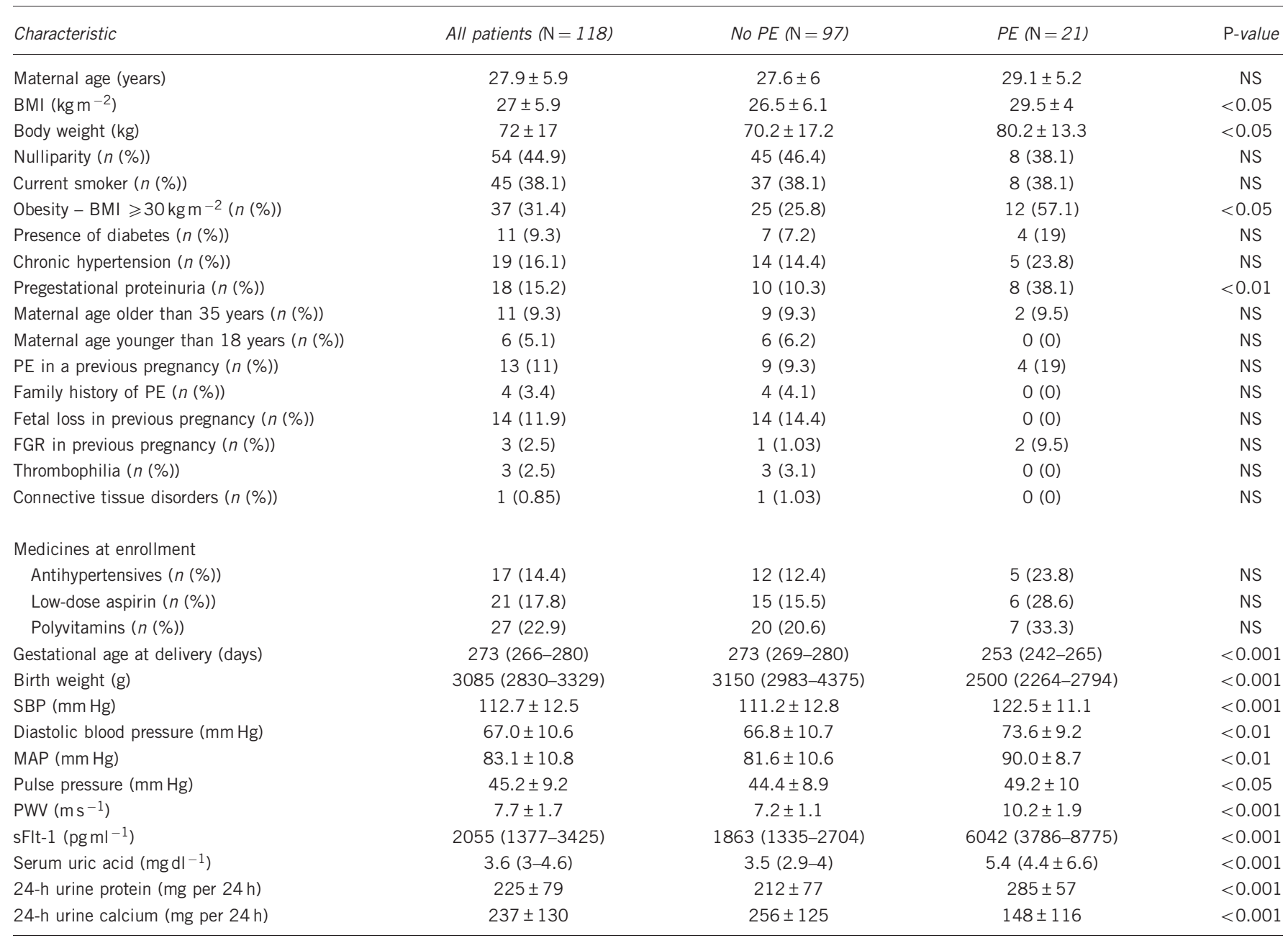

Abbreviations: BMI, body mass index; FGR, fetal growth restriction; MAP, mean arterial pressure; NS, not significant; PWV, pulse wave velocity; SBP, systolic blood pressure; sFlt-1, soluble fms-like tyrosine kinase 1 .

Data are expressed as mean \pm s.d. or as median (interquartile range).

was set (a) at $90 \%$ sensitivity by ROC analysis, thus enabling comparisons of the false-positive rate (FPR) for each factor at an equivalent sensitivity and (b) at 10\% FPR to enable comparison of the sensitivity at an equivalent FPR. $P$-values of $<0.05$ (two-tailed) were considered significant.

\section{RESULTS}

Of 138 patients meeting inclusion criteria, 132 (96\%) agreed to participate and were enrolled in the study. Of these individuals, 14 were excluded due to a lack of pregnancy outcome data. Among the 118 cases included in the analyses, 21 developed PE. Of these patients, 11 developed early-onset PE (diagnosis at $<34$ weeks) and 10 developed late-onset PE (diagnosis at 34 weeks or later). The baseline characteristics of pre-eclamptic and non-pre-eclamptic women are shown in Table 1. PE was more frequent among women who were overweight (BMI $\geq 30 \mathrm{~kg} \mathrm{~m}^{-2}$ ) and those with pregestational proteinuria. There were no differences between the two groups in maternal age, nulliparity, chronic hypertension, diabetes, young ( $<18$ years) or old ( $>35$ years) maternal age, smoking habits, $\mathrm{PE}$ in a previous pregnancy, family history of $\mathrm{PE}$, fetal demise in a previous pregnancy, thrombophilia, connective tissue disorders, fetal growth restriction in a previous pregnancy or medicine use (antihypertensives—-such as methyldopa, nifedipine and beta-blockers—, low-dose aspirin: $80-100 \mathrm{mg}$ daily and polyvitamins including folate). Compared with non-pre-eclamptic women, pre-eclamptic women showed significantly higher $\left(10.2 \pm 1.9\right.$ vs. $\left.7.2 \pm 1.1 \mathrm{~ms}^{-1} ; P<0.001\right) \mathrm{PWV}$ (Figure 2), blood pressure (systolic, diastolic and mean pressure), serum sFlt-1 (Figure 2), serum uric acid, 24-h urine protein excretion and lower 24-h urinary calcium excretion, maternal age at delivery and birth weight babies. PWV measurements were not affected by antihypertensive medications because the difference in PWV between pre-eclamptic and normotensive women remained similar after excluding medicated hypertensive women $(10.3 \pm 2.0$ vs. $\left.7.1 \pm 1.1 \mathrm{~m} \mathrm{~s}^{-1} ; P<0.001\right)$. Notably, PWV was greater $(8.2 \pm 1.8 v s$. $\left.7.1 \pm 1.3 \mathrm{~m} \mathrm{~s}^{-1} ; P<0.001\right)$ in 69 women with risk factors that are known to be associated with increased AS, such as pregestational diabetes mellitus, obesity, chronic hypertension, proteinuria, old maternal age, prior history of $\mathrm{PE}$ and connective tissue diseases compared with 49 women with risk factors unrelated to AS, such as nulliparity, young maternal age, family history of PE, thrombophilia and fetal growth restriction or fetal demise in a previous pregnancy. Finally, subjects with early-onset PE compared with those with late-onset PE showed significantly higher PWV and mean arterial pressure and lower pre-pregnancy BMI, maternal age, gestational age at delivery and birth weight of infants (Table 2). None of the other 

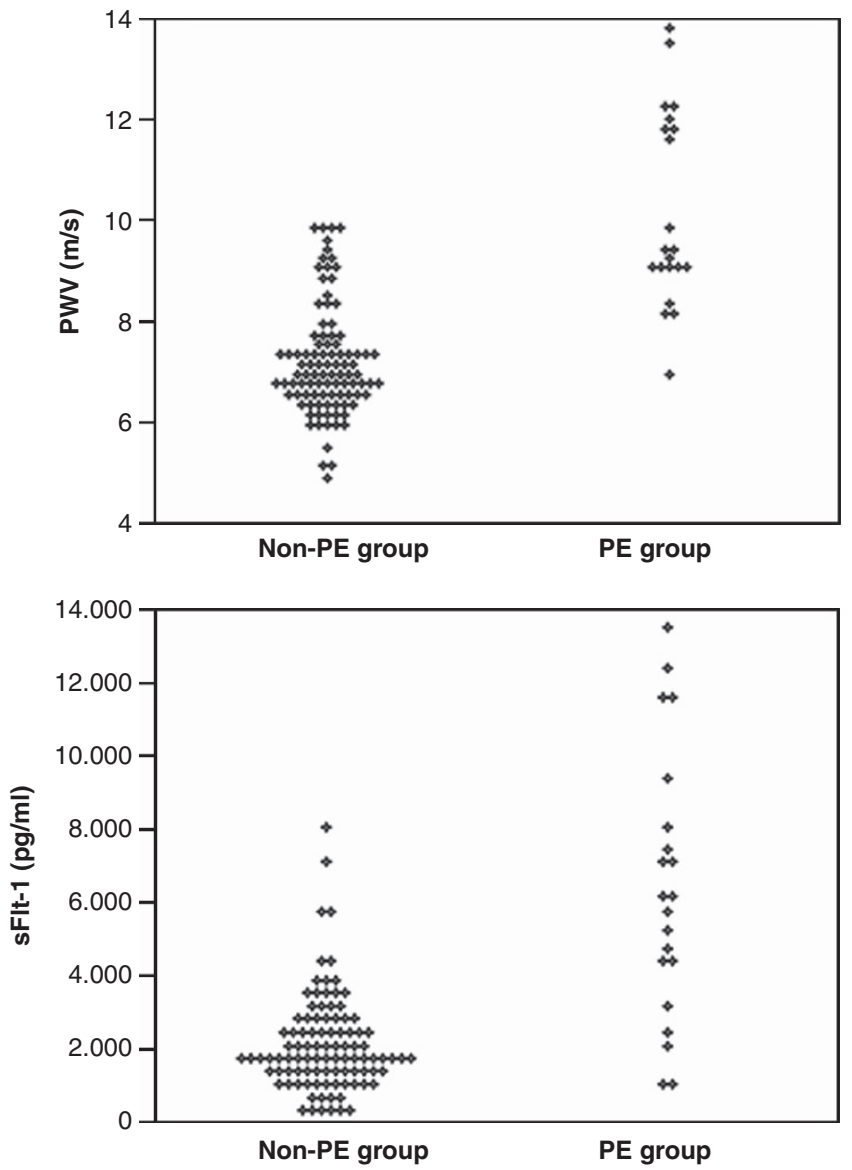

Figure 2 PWV $\left(\mathrm{m} \mathrm{s}^{-1}\right)$ and sFlt-1 $\left(\mathrm{pg} \mathrm{ml}^{-1}\right)$ at 22-26 weeks of gestation in the non-PE and PE groups.

parameters measured at enrollment differed between early- and lateonset PE.

As shown in Table 3, PWV was associated with more risk factors and markers for PE than sFlt-1 and the associations were generally stronger. On univariate analysis, PWV was positively associated with sFlt-1 $(r=0.408 ; P<0.001)$, age, blood pressure, BMI, diabetes, serum uric acid and 24-h urine protein excretion, whereas it was inversely related to 24 -h calcium excretion. The results of a forward stepwise multivariate analysis showed that SBP, sFlt-1, serum uric acid, age and 24-h urine protein were independent determinants of PWV (Table 4). Together, these five variables explained $41.6 \%$ of the variance in PWV.

ROC curves were constructed for each marker, and the area under each curve was calculated (Table 5). PWV showed the best predictive performance for all cases of $\mathrm{PE}$ and for early-onset $\mathrm{PE}$ as it yielded the highest area under the curve values of 0.921 and 0.948 , respectively, followed by sFlt-1, uric acid, 24-h urinary protein and calcium excretion. Of the different combinations of predictive tests performed, the best results for all PE and early-onset PE were obtained when PWV was combined with sFlt-1, where area under the curve values increased to 0.965 and 0.963 , respectively (Figure 3). Despite the limited ability of PWV to predict late-onset PE, when combined with sFlt-1 its predictive performance for the disease improved significantly (area under the curve increased from 0.806 to $0.871 ; P<0.05$ ). The predictive performance of serum uric acid, 24-h urine protein and calcium was inferior to that of PWV and sFlt-1; thus, they will not be further discussed as predictors of PE.
Next, for each marker, the sensitivity at a fixed FPR of $10 \%$ and the FPR at a fixed sensitivity of $90 \%$ were calculated using ROC analysis (Table 5). PWV showed the highest detection rate of all PE (81\%) and early-onset PE $(82 \%)$ at a fixed $10 \%$ FPR, and when combined with sFlt-1, these rates increased to $90 \%$ and $92 \%$, respectively. The combination of PWV with sFlt-1 achieved a detection rate of $50 \%$ for late-onset $\mathrm{PE}$ at a fixed $10 \% \mathrm{FPR}$, which was 2.5 -fold greater $(P<0.05)$ than that of PWV $(20 \%)$ alone. PWV showed the lowest FPR for all PE (17\%) and early-onset PE (14\%) at a fixed $90 \%$ sensitivity. The FPR was further decreased (9\%) for both conditions when PWV was combined with sFlt-1.

Next, we examined whether PWV and sFlt-1 impacted the development of PE independently of each other. A multivariate (mean arterial pressure, BMI, age) adjusted logistic regression analysis demonstrated a 3.9 -fold (95\% CI 2.2-7) increase in risk for all PE for every $1 \mathrm{~m} \mathrm{~s}^{-1}$ increase in PWV and a 6.5-fold (95\% CI 2.2-19.2) increase in risk for every 1 s.d. of log-transformed sFlt-1, confirming that PWV and sFlt-1 were independent predictors of all PE.

Finally, based on ROC analysis, a cutoff value of $8 \mathrm{~m} \mathrm{~s}^{-1}$ for PWV resulted in the greatest sensitivity and specificity for predicting all PE (95\% and $82 \%$, respectively), whereas a suggested PWV cutoff value of $9 \mathrm{~m} \mathrm{~s}^{-1}$ predicted early-onset PE with a sensitivity and specificity of $91 \%$ and $86 \%$, respectively.

\section{DISCUSSION}

The results of this study showed that maternal AS, as assessed by PWV during the second trimester, was $30 \%$ greater in women who subsequently developed PE compared with normotensive controls. Of the five markers tested, PWV showed the highest diagnostic power for all PE and early-onset PE but not for late-onset PE. The combination of PWV with sFlt-1 further improved the prediction of all subtypes of PE.

Recently, it has become evident that aberrant hemodynamic adaptations in PE also include AS of the larger elastic arteries in addition to high resistance in small peripheral arteries. ${ }^{21} \mathrm{PWV}$, by directly reflecting large artery stiffness and having the greatest predictive value for cardiovascular outcomes, ${ }^{13}$ is now considered the gold standard for assessing AS in daily practice. ${ }^{18}$ Maternal AS, as assessed by PWV, at a pre-pregnant state (follicular phase), ${ }^{22} 11-13$ and 22-24 weeks of gestation ${ }^{23,24}$ and 4 months to 2 years postpartum, ${ }^{25,26}$ was consistently increased in pre-eclamptic compared with non-pre-eclamptic women. The findings of this current study confirm the results of this previously reported association between PWV and PE and further extend these finding to women at high risk for PE, where this association may be more pronounced. We show that PWV increased by $30 \%$ in women destined to develop PE compared with normotensive women. The magnitude of increase in PWV during the late second trimester was greater than that reported $^{24,27}$ in women at $22-24$ weeks of gestation $(17 \%)$ or in women with established PE (18\%). The fact that a high percentage $(58.5 \%)$ of pregnant women in our study showed pre-existing medical conditions associated with the risk of endothelial dysfunction and AS may underlie the greater increase in PWV among women with $\mathrm{PE}$ than those without PE when compared with other previous studies. These findings suggest that pre-existing maternal subclinical endothelial dysfunction and atherosclerosis may render pregnant women more sensitive to maladaptive hemodynamic responses including increased AS, and thus placing them at high risk for developing PE. 
Table 2 Baseline characteristics of the two PE groups

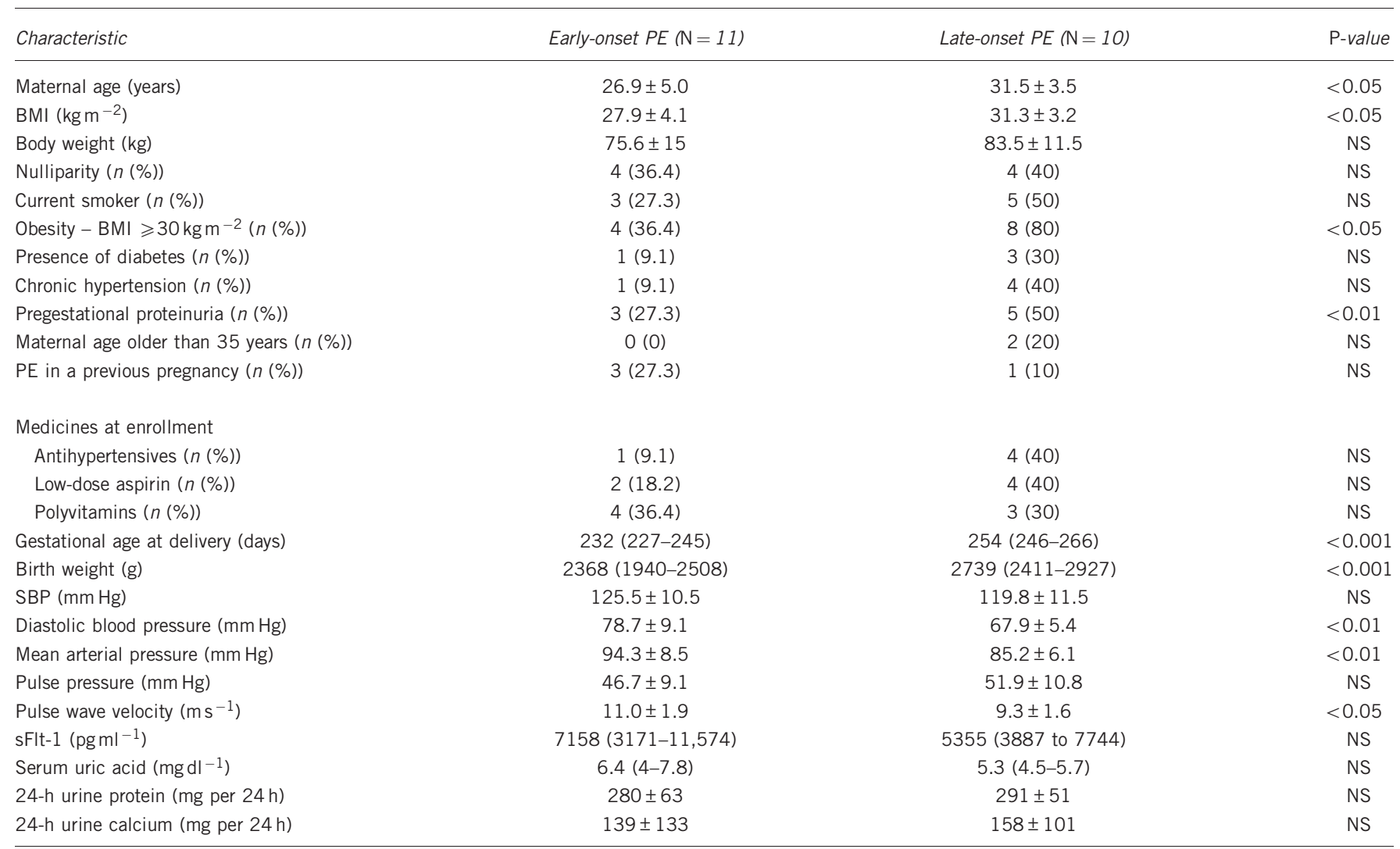

Abbreviations: BMI, body mass index; FGR, fetal growth restriction; NS, not significant; SBP, systolic blood pressure; sFlt-1, soluble fms-like tyrosine kinase 1 .

Data are expressed as mean \pm s.d. or as median (interquartile range).

Table 3 Correlations of carotid-femoral PWV and soluble fms-like tyrosine kinase 1 (sFlt-1) with selected maternal demographic, clinical, hemodynamic and biochemical characteristics at study entry in 118 high-risk pregnant women

\begin{tabular}{|c|c|c|c|c|}
\hline \multirow[b]{2}{*}{ Parameter } & \multicolumn{2}{|c|}{$P W V$} & \multicolumn{2}{|c|}{$s F / t-l \log$} \\
\hline & $r$ & P-value & $r$ & P-value \\
\hline Age & 0.283 & $<0.01$ & -0.033 & NS \\
\hline Body weight & 0.313 & $<0.001$ & -0.025 & NS \\
\hline BMI & 0.271 & $<0.01$ & -0.005 & NS \\
\hline Diabetes & 0.219 & $<0.05$ & 0.027 & NS \\
\hline PWV & & & 0.408 & $<0.001$ \\
\hline SBP & 0.452 & $<0.001$ & 0.274 & $<0.01$ \\
\hline DBP & 0.288 & $<0.01$ & 0.165 & NS \\
\hline MAP & 0.376 & $<0.001$ & 0.222 & $<0.05$ \\
\hline Pulse pressure & 0.314 & $<0.01$ & 0.201 & $<0.05$ \\
\hline sFlt- $1_{\log }$ & 0.408 & $<0.001$ & & \\
\hline Serum uric acid $\log$ & 0.433 & $<0.001$ & 0.240 & $<0.01$ \\
\hline 24-h urine protein & 0.303 & $<0.01$ & 0.185 & $<0.05$ \\
\hline 24-h urine calcium & -0.279 & $<0.01$ & -0.199 & $<0.05$ \\
\hline Birth weight & -0.490 & $<0.001$ & -0.335 & $<0.001$ \\
\hline GA at delivery & -0.580 & $<0.001$ & -0.435 & $<0.001$ \\
\hline
\end{tabular}

Abbreviations: DBP, diastolic blood pressure; GA, gestational age; MAP, mean arterial pressure; $r$, Pearson correlation coefficient; SBP, systolic blood pressure.

In this current study, a strong correlation was observed in univariate analysis between PWV and sFlt-1 $(r=0.408 ; P<0.001)$, whereas in our multivariate adjusted regression model, sFlt-1 emerged
Table 4 Multivariate regression analysis for assessing the predictors of PWV

\begin{tabular}{lcccccc}
\hline Parameter & B & $\begin{array}{c}\text { Standard } \\
\text { error }\end{array}$ & $\begin{array}{c}\text { Standard } \\
\text { beta }\end{array}$ & $\begin{array}{c}\text { P- } \\
\text { value }\end{array}$ & $\begin{array}{c}\text { Partial } \\
\mathrm{r}\end{array}$ & $\begin{array}{c}\text { Adjusted } \\
\mathrm{R}^{2} \text { changes }\end{array}$ \\
\hline constant & -4.232 & 1.464 & & 0.005 & & \\
SBP & 0.032 & 0.010 & 0.249 & 0.002 & 0.288 & 0.197 \\
sFIt- $\log _{\log }$ & 1,221 & 0.373 & 0.249 & 0.001 & 0.296 & 0.279 \\
Uric acid & 2,833 & 0.849 & 0.255 & 0.001 & 0.301 & 0.337 \\
Age & 0.060 & 0.021 & 0.204 & 0.006 & 0.256 & 0.384 \\
24-h UP & 0.004 & 0.002 & 0.195 & 0.008 & 0.246 & 0.416 \\
\hline
\end{tabular}

Abbreviations: PWV, pulse wave velocity; $r$, correlation coefficient; SBP, systolic blood pressure; sFlt-1, soluble fms-like tyrosine kinase 1 ; standard beta, standardized regression coefficients; SFIt-1, soluble fms-ik, urine protein.
UP,

as a major determinant of PWV, contributing to $\sim 8 \%$ of its variability. This strong dependence of PWV on sFlt-1, an antiangiogenic factor that impairs endothelial function by reducing nitric oxide synthesis, ${ }^{28-30}$ further confirms the well-documented association between endothelial dysfunction and increased large artery stiffness in non-pregnant populations. ${ }^{31-33}$ SBP, another factor that causes functional stiffening of the large arteries by distending the arterial walls, emerged as the strongest predictor of PWV, explaining $\sim 20 \%$ of the variation in PWV. These findings show clearly that blood pressure modulates AS independently of mediators affecting smooth muscle tone. Finally, whether an alteration in structural elements (elastin, collagen) in the arterial wall contributes to the increased AS in PE is far from clear. Although signs of remodeling in 


\begin{tabular}{|c|c|c|c|c|c|c|}
\hline \multirow[b]{2}{*}{ Marker } & \multirow[b]{2}{*}{$A \cup C$} & \multirow[b]{2}{*}{$(95 \% \mathrm{Cl})$} & \multicolumn{2}{|c|}{ FPR at $10 \%$} & \multicolumn{2}{|c|}{ Sensitivity at $90 \%$} \\
\hline & & & Sensitivity & $(95 \% \mathrm{Cl})$ & $F P R$ & $(95 \% \mathrm{Cl})$ \\
\hline \multicolumn{7}{|l|}{$A \| l P E(\mathrm{n}=21)$} \\
\hline PWV $\left(\mathrm{ms}^{-1}\right)$ & 0.921 & $0.856-0.962$ & 81 & $58-95$ & 17 & $11-26$ \\
\hline sFlt-1 (pg ml-1) & 0.865 & $0.790-0.921$ & 76 & $53-92$ & 43 & $34-55$ \\
\hline PWV $\left(\mathrm{ms}^{-1}\right)+\mathrm{sFlt}-1\left(\mathrm{pg} \mathrm{ml}^{-1}\right)$ & 0.965 & $0.913-0.990$ & 90 & 70-99 & 9 & $4-17$ \\
\hline Serum uric acid $\left(\mathrm{mg} \mathrm{dl}^{-1}\right)$ & 0.871 & $0.796-0.925$ & 62 & $38-82$ & 31 & $22-41$ \\
\hline Urine protein excretion (mg per $24 \mathrm{~h}$ ) & 0.811 & $0.729-0.877$ & 29 & $11-52$ & 30 & $21-40$ \\
\hline Urine Ca excretion (mg per $24 \mathrm{~h}$ ) & 0.775 & $0.654-0.847$ & 48 & $26-70$ & 52 & $41-62$ \\
\hline \multicolumn{7}{|l|}{ Early-onset PE $(\mathrm{n}=11)$} \\
\hline $\mathrm{PWV}\left(\mathrm{ms}^{-1}\right)$ & 0.948 & 0.899-0.997 & 82 & $48-98$ & 14 & $8-22$ \\
\hline sFlt-1 (pg ml-1) & 0.836 & $0.757-0.898$ & 73 & 39-94 & 48 & $38-58$ \\
\hline $\mathrm{PWV}\left(\mathrm{ms}^{-1}\right)+\mathrm{sFlt} 1\left(\mathrm{pg} \mathrm{ml}^{-1}\right)$ & 0.963 & $0.922-0.999$ & 92 & $59-99$ & 9 & $5-17$ \\
\hline Serum uric acid $\left(\mathrm{mg} \mathrm{dl}^{-1}\right)$ & 0.835 & $0.755-0.897$ & 55 & $23-83$ & 36 & $27-46$ \\
\hline Urine protein excretion (mg per $24 \mathrm{~h}$ ) & 0.760 & $0.673-0.834$ & 10 & $0.2-41$ & 35 & $26-44$ \\
\hline Urine Ca excretion (mg per $24 \mathrm{~h}$ ) & 0.786 & $0.701-0.856$ & 36 & $11-69$ & 31 & $22-41$ \\
\hline \multicolumn{7}{|l|}{ Late-onset PE $(\mathrm{n}=10)$} \\
\hline $\mathrm{PWV}\left(\mathrm{ms}^{-1}\right)$ & 0.806 & $0.723-0.873$ & 20 & $3-56$ & 25 & $17-34$ \\
\hline sFlt-1 (pg ml-1) & 0.822 & $0.741-0.886$ & 50 & $19-81$ & 40 & $31-51$ \\
\hline $\mathrm{PWV}\left(\mathrm{ms}^{-1}\right)+\mathrm{sFlt} 1\left(\mathrm{pg} \mathrm{ml}^{-1}\right)$ & 0.871 & $0.797-0.926$ & 50 & $19-81$ & 30 & $21-39$ \\
\hline Serum uric acid $\left(\mathrm{mg} \mathrm{dl}^{-1}\right)$ & 0.834 & $0.755-0.896$ & 10 & $0.3-45$ & 24 & $16-33$ \\
\hline Urine protein excretion (mg per $24 \mathrm{~h}$ ) & 0.803 & $0.720-0.871$ & 30 & $8-65$ & 36 & $27-44$ \\
\hline Urine Ca excretion (mg per 24 h) & 0.706 & $0.616-0.787$ & 40 & $12-74$ & 56 & $45-66$ \\
\hline
\end{tabular}

Abbreviations: AUC, area under the curve; FPR, false positive rate; PWV, pulse wave velocity; sFlt-1, soluble fms-like tyrosine kinase 1.

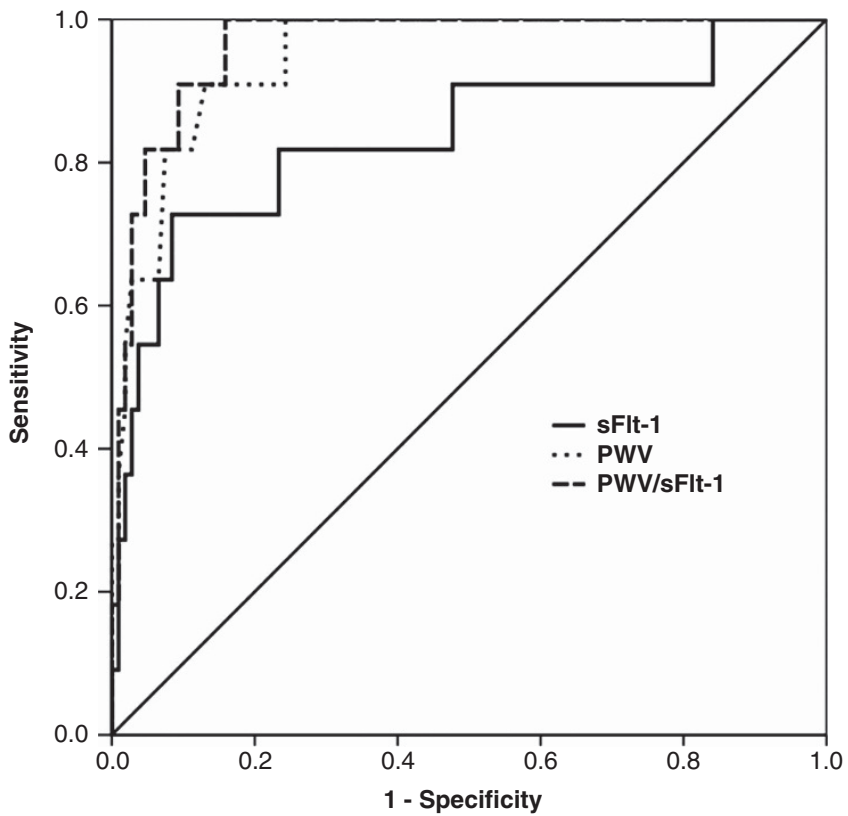

Figure 3 Receiver operating characteristic curves depicting the sensitivity and specificity of pulse wave velocity, sFIt-1 and their combination in identifying all cases of PE.

vascular beds ${ }^{34}$ and carotid intima-media thickness ${ }^{35}$ and data linking matrix metalloproteinases to cardiac/artery remodeling in $\mathrm{PE}^{36}$ have been reported, any major changes in the elastic properties of the aortic walls are unlikely to develop during the short duration of the disease. Therefore, factors linked with functional rather than structural changes of the endothelium or the medial layer appear to cause the changes in AS during pregnancy.

AS may also be influenced by other conventional risk factors that affect vascular function. ${ }^{37,38}$ As expected, the most important, age and blood pressure, ${ }^{38}$ were also identified as major determinants of AS in this study. In this context, uric acid emerged as a major determinant of PWV, explaining $6 \%$ of its variability and further confirming the uric acid-PWV association previously reported in non-pregnant populations. ${ }^{39,40}$ Uric acid, which is now thought to increase not only due to decreased clearance but also via increased uric acid production during pregnancy due to trophoblast breakdown, cytokine release and ischemia, can promote inflammation, ${ }^{41}$ endothelial dysfunction and oxidation, ${ }^{42}$ factors known to contribute to arterial stiffening. Finally, the endothelial dysfunction, caused by an imbalance of pro- and anti-angiogenic proteins, is undoubtedly the mechanism underlying the observed association ${ }^{43}$ between proteinuria and PWV, the existence of which has also been well documented in healthy individuals ${ }^{44}$ and selected patients groups. ${ }^{45}$ Taken together, all these data indicate that cardiovascular risk factors thought to exert an important influence on AS in non-pregnant populations are also predictive in pregnancy. Thus, for a wider implementation of PWV for prediction of PE, the influence of other cardiovascular risk factors on PWV should be taken into account.

To the best of our knowledge, this study is the first to evaluate $\mathrm{PWV}$ as a predictor of PE in high-risk women. On the basis of our results, PWV appeared to be a valuable predictor of $\mathrm{PE}$, particularly early-onset $\mathrm{PE}$. In populations of low-risk pregnant women, $\mathrm{PWV}^{23}$ was a significant predictor of PE (OR 44.5 (95\% CI, 5.7-348.6) at 11-13 weeks of gestation, and the augmentation index, a measure of wave reflection and AS, showed a detection rate of $79 \%$ for all cases of 
PE and $88 \%$ for early-onset PE for a FPR of $11 \% .{ }^{46}$ By contrast, PWV emerged as a poor predictor of late-onset PE. These findings confirm the results of previous studies, where a significantly higher augmentation index was reported in early-onset PE compared with late-onset PE when patients were studied during pregnancy, ${ }^{47}$ and in women with previous early-onset PE compared with women with previous late-onset PE. ${ }^{48}$ In our study, PWV was significantly higher in the early-onset $\mathrm{PE}$ group compared with the late-onset $\mathrm{PE}$ group $\left(11 \pm 1.9\right.$ vs. $\left.9.3 \pm 1.6 \mathrm{~m} \mathrm{~s}^{-1} ; P<0.05\right)$. These differences may simply reflect the fact that early- and late-onset PE are part of a wide spectrum of the disease, with early-onset PE being the most severe of the spectrum. However, the association of a lower newborn birth weight with early-onset PE and the association of an older maternal age and higher BMI with late-onset PE are findings consistent with previous epidemiologic studies ${ }^{4-51}$ and that are compatible with the concept that early- and late-onset PE may be two different disorders where early-onset PE is related to reduced placental perfusion and late-onset $\mathrm{PE}$ is associated with maternal factors. Irrespective of whether PE is one disease of varying degrees of severity or is separated into two distinct pathophysiologic entities, ${ }^{50,52}$ the fact that PWV appears to be a much better predictor of early-onset than late-onset $\mathrm{PE}$ is of clinical relevance because it is the early rather than late disease that is associated with an increased risk of perinatal morbidity and mortality and both short-term and long-term maternal complications. ${ }^{53,54}$

In an effort to further improve disease prediction, PWV was investigated in combination with other markers. The predictive performance of PWV improved only when it was combined with sFlt-1. Given the complex pathophysiology underlying PE, the combination of two or more independent biomarkers, each reflecting a different pathophysiological process, generally lead to an improved performance for the early prediction of $\mathrm{PE}$ compared with single markers. ${ }^{12}$ Thus, this study provides evidence that the increased AS (PWV) and endothelial dysfunction due to impaired nitric oxide production (sFlt-1), irrespective of any interrelationship, are highly likely to reflect diverse pathogenic processes in the development of $\mathrm{PE}$. The above theory is further supported by our findings that (a) the associations of selected maternal factors with PWV differed to those with sFlt-1, both qualitatively and quantitatively (Table 2), and (b) the risk of subsequent development of PE was predicted independently by both PWV and sFlt-1 after adjusting for potential confounding factors.

This study has several limitations. The sample size of this study was small, with only 11 and 10 patients developing early- and late onset $\mathrm{PE}$, respectively. Nevertheless, the prevalence of $\mathrm{PE}$ in this study was high (18\%), which may be explained by the high-risk nature of our study population. Given that all women participating in the study were at high risk for developing PE, it is likely that the PWV and the other screening markers described in this study would not perform as well in women at low risk of developing PE. Furthermore, PWV may show optimal performance in predicting PE in the presence of risk factors related to subclinical vascular dysfunction, as in this study, and reduced diagnostic performance in their absence. In a recent study ${ }^{55}$ of 180 pregnant women with $\geqslant 2$ risk factors for $\mathrm{PE}$, the augmentation index, a marker of wave reflection and AS, did not provide additional information beyond brachial blood pressure and maternal risk factor profile on the risk of development of future PE. However, women with chronic hypertension, diabetes and renal disease (presumed preexisting vascular abnormalities) were excluded from the study, which may explain why this indirect marker of AS failed to predict PE in women at high risk. Apart from the absence of women at low risk for $\mathrm{PE}$, the lack of other ethnic groups and the fact that a single center participated in this study could also jeopardize the generalizability of the study. Therefore, larger multicenter studies will be required to establish the accuracy of PWV for PE prediction in low- and high-risk populations. Despite these limitations, this study enabled us to detect robust correlations between PWV and mediators of endothelial cell dysfunction (sFlt-1, uric acid) in our high-risk pregnant women, which may help to further elucidate the mechanisms underlying the association between PE and AS.

Although no intervention has yet proven effective for the prevention of PE, early identification of women at risk for PE may improve maternal and perinatal outcome. Screening for PE is believed to be most relevant during the first trimester because preventive interventions (such as antiplatelet agents, calcium and antioxidants) are more likely to be effective if initiated early in pregnancy when pathogenic mechanisms may be modified. Further confirmation of the risk of future PE based on sFlt-1 levels ${ }^{11}$ and, potentially, PWV measurements in the second trimester may enable closer prenatal monitoring, earlier diagnosis and prompt and appropriate management (antenatal corticosteroids for fetal lung maturation, treatment of severe hypertension, magnesium for seizure prophylaxis, bed rest or early delivery). The potential benefits of screening for PWV may be higher in pregnant women at high baseline risk for PE based on underlying maternal vascular disease. Thus, predicting PE in women with underlying conditions including diabetes and chronic hypertension would be of great clinical value. Sequential measurements of markers may also improve risk assessment because changes in individual markers from the first to second trimester may herald the onset of PE. Indeed, longitudinal studies suggest that the importance of the association between AS and PE lies in the temporal changes in AS measurements throughout pregnancy, ${ }^{15,56}$ whereas sequential changes in antiangiogenic factors, such as sFlt-1, during pregnancy were shown to be predictive of preterm $\mathrm{PE}^{57}$ or overall $\mathrm{PE}$ risk. $^{58}$

In summary, PWV, a simple, low-cost noninvasive method for assessing AS, measured during the second trimester, may prove useful in predicting $\mathrm{PE}$, particularly early-onset $\mathrm{PE}$, in high-risk women. The predictive characteristics of PWV were further improved when it was used in concert with sFlt-1. The extent to which PWV alone or in combination with other biomarkers is useful in screening for $\mathrm{PE}$ remains to be determined.

1 Noris M, Perico N, Remuzzi G. Mechanisms of disease: preeclampsia. Nat Clin Pract Nephrol 2005; 1: 98-114.

2 Roberts JM, Gammil HS. Preeclampsia: recent insights. Hypertension 2005; 46: 1243-1249.

3 Hladunewich M, Karumanchi SA, Lafayette R. Pathophysiology of the clinical manifestations of preeclampsia. Clin J Am Soc Nephrol 2007; 2: 543-549.

4 Wang A, Rana S, Karumanchi SA. Preeclampsia: the role of angiogenic factors in pathogenesis. Physiology (Bethesda) 2009; 24: 147-158.

5 Maynard SE, Thadhani R. Pregnancy and the kidney. J Am Soc Nephrol 2009; 20 : 14-22.

6 Levine RJ, Maynard SE, Qian C, Lim KH, England LJ, Yu KF, Schisterman EF, Thadhani R, Sachs BP, Epstein FH, Sibai BM, Sukhatme VP, Karumanchi SA. Circulating angiogenic factors and the risk of preeclampsia. N Engl J Med 2004; 350: 672-683.

7 Maynard SE, Min J-Y, Merchan J, Lim K-H, Li J, Mondal S, Libermann TA, Morgan JP, Sellke FW, Stillman IE, Epstein FH, Sukhatme VP, Karumanchi SA. Excess placental soluble fms-like tyrosine kinase 1 (sflt1) may contribute to endothelial dysfunction, hypertension, and proteinuria in preeclampsia. J Clin Invest 2003; 111: 649-658.

8 Conde-Agudelo A, Villar J, Lindheimer M. World Health Organization systematic review of screening tests for preeclampsia. Obstet Gynecol 2004; 104: 1367-1391.

9 Cnossen JS, ter Riet G, Mol BW, van der Post JA, Leeflang MM, Meads CA, Hyde C, Khan KS. Are tests for predicting pre-eclampsia good enough to make screening viable? A review of reviews and critical appraisal. Acta Obstet Gynecol Scand 2009; 88: 758-765. 
10 Meads CA, Cnossen JS, Meher S, Juarez-Garcia A, ter Riet G, Duley L, Roberts TE, Mol BW, van der Post JA, Leeflang MM, Barton PM, Hyde CJ, Gupta JK, Khan KS Methods of prediction and prevention of pre-eclampsia: systematic reviews of accuracy and effectiveness literature with economic modelling. Health Technol Assess 2008, 12: iii-iv.

11 Kleinrouweler CE, Wiegerinck MM, Ris-Stalpers C, Bossuyt PM, van der Post JA von Dadelszen P, Mol BW, Pajkrt E; EBM CONNECT Collaboration. Accuracy of circulating placental growth factor, vascular endothelial growth factor, soluble fms-like tyrosine kinase 1 and soluble endoglin in the prediction of pre-eclampsia: a systematic review and meta-analysis. BJOG 2012; 119: 778-787.

12 Giguère $Y$, Charland $M$, Bujold $E$, Bernard N, Grenier $S$, Rousseau $F$, Lafond J, Légaré $F$, Forest JC. Combining biochemical and ultrasonographic markers in predicting preeclampsia: a systematic review. Clin Chem 2010; 56: 361-371.

13 Mitchell GF, Hwang SJ, Vasan RS, Larson MG, Pencina MJ, Hamburg NM, Vita JA, Levy D, Benjamin EJ. Arterial stiffness and cardiovascular event. The Framingham Heart Study. Circulation 2010; 121: 505-511.

14 Hausvater A, Giannone T, Sandoval YH, Doonan RJ, Antonopoulos CN, Matsoukis IL Petridou ET, Daskalopoulou SS. The association between preeclampsia and arterial stiffness. J Hypertens 2012; 30: 17-33.

15 Oyama-Kato M, Ohmichi M, Takahashi K, Suzuki S, Henmi N, Yokoyama Y, Kurachi H. Change in pulse wave velocity throughout normal pregnancy and its value in predicting pregnancy-induced hypertension: a longitudinal study. Am J Obstet Gynecol 2006; 195: 464-469.

16 Khalil A, Jauniaux E, Cooper D, Harrington K. Pulse wave analysis in normal pregnancy: a prospective longitudinal study. PLoS One 2009; 4: e6134.

17 Laurent S, Cockcroft J, Van Bortel L, Boutouyrie P, Giannattasio C, Hayoz D, Pannier B, Vlachopoulos C, Wilkinson I, Struijker-Boudier H; European Network for Non-invasive Investigation of Large Arteries. Expert consensus document on arterial stiffness: methodological issues and clinical applications. Heart J 2006; 27: 2588-2605.

18 Van Bortel LM, Laurent S, Boutouyrie P, Chowienczyk P, Cruickshank JK, De Backer T, Filipovsky J, Huybrechts S, Mattace-Raso FU, Protogerou AD, Schillaci G, Segers P, Vermeersch S, Weber TArtery Society; European Society of Hypertension Working Group on Vascular Structure and Function; European Network for Noninvasive Investigation of Large Arteries. Expert consensus document on the measurement of aortic stiffness in daily practice using carotid-femoral pulse wave velocity. J Hypertens 2012; 30: 445-448.

19 Report of the National High Blood Pressure Education Program Working Group on High Pressure in Pregnancy. Am J Obstet Gynaecol 2000; 183: S1-S22.

20 Sollinger DF, Wilhelm A, Uehlinger D, Frey FJ. Arterial stiffness assessed by digital volume pulse correlates with comorbidity in patients with ESRD. Am J Kidney Dis 2006; 48: 456-463.

21 Tihtonen KM, Kööbi T, Uotila JT. Arterial stiffness in preeclamptic and chronic hypertensive pregnancy. Eur J Obstet Gynecol Reprod Biol 2006; 128: 180-186.

22 Hale SA, Badger GJ, McBride C, Magness R, Bernstein IM. Prepregnancy vascular dysfunction in women who subsequently develop hypertension during pregnancy. Pregnancy Hypertens 2013; 3: 140-145.

23 Khalil A, Akolekar R, Syngelaki A, Elkhouli M, Nicolaides KH. Maternal hemodynamics at 11-13 weeks' gestation and risk of pre-eclampsia. Ultrasound Obstet Gynecol 2012; 40: 28-34.

24 Savvidou MD, Kaihura C, Anderson JM, Nicolaides KH. Maternal arterial stiffness in women who subsequently develop pre-eclampsia. PLoS One 2011; 6: e18703.

25 Elvan-Taspinar A, Bots ML, Franx A, Bruinse HW, Engelbert RH. Stiffness of the arterial wall, joints and skin in women with a history of preeclampsia. J Hypertens 2005; 23 $147-151$

26 Pàez O, Alfie J, Gorosito M, Puleio P, de Maria M, Prieto N, Majul C. Parallel decrease in arterial distensibility and in endothelium-dependent dilatation in young women with a history of pre-eclampsia. Clin Exp Hypertens 2009; 31: 544-552.

27 Kaihura C, Savvidou MD, Anderson JM, McEniery CM, Nicolaides KH. Maternal arterial stiffness in pregnancies affected by preeclampsia. Am J Physiol Heart Circ Physiol 2009; 297: H759-H764.

28 Di Marco GS, Reuter S, Hillebrand U, Amler S, König M, Larger E, Oberleithner H, Brand $\mathrm{E}$, Pavenstädt $\mathrm{H}$, Brand $\mathrm{M}$. The soluble VEGF receptor sFlt1 contributes to endothelial dysfunction in CKD. J Am Soc Nephrol 2009; 20: 2235-2245.

29 Dimmeler S, Dernbach E, Zeiher AM. Phosphorylation of the endothelial nitric oxide synthase at ser-1177 is required for VEGF-induced endothelial cell migration. FEBS Lett 2000; 477: 258-262.

30 Sandrim VC, Palei AC, Metzger IF, Gomes VA, Cavalli RC, Tanus-Santos JE. Nitric oxide formation is inversely related to serum levels of antiangiogenic factors soluble fms-liketyrosine kinase-1 and soluble endogline in preeclampsia. Hypertension 2008; 52: 402-407.

31 McEniery CM, Wallace S, Mackenzie IS, McDonnell B, Yasmin, Newby DE, Cockcroft JR, Wilkinson IB. Endothelial function is associated with pulse pressure, pulse wave velocity, and augmentation index in healthy humans. Hypertension 2006; 48: 602-608

32 Ravikumar R, Deepa R, Shanthirani C, Mohan V. Comparison of carotid intima-media thickness, arterial stiffness, and brachial artery flow mediated dilatation in diabetic and nondiabetic subjects. Am J Cardiol 2002; 90: 702-707.
33 Nigam A, Mitchell GF, Lambert J, Tardif JC. Relation between conduit vessel stiffness (assessed by tonometry) and endothelial function (assessed by flow-mediated dilatation) in patients with and without coronary heart disease. Am J Cardiol 2003; 92: 395-399.

34 Aalkjaer C, Johannesen P, Petersen EB, Rasmussen A, Mulvany MJ. Characteristics of resistance vessels in pre-eclampsia and normotensive pregnancy. J Hypertens Suppl 1984; 2: S183-S185.

35 Stergiotou I, Crispi F, Valenzuela-Alcaraz B, Bijnens B, Gratacos E. Patterns of maternal vascular remodeling and responsiveness in early versus late onset preeclampsia. Am J Obstet Gynecol 2013; 209: 558.e1-558.e14.

36 Palei AC, Granger JP, Tanus-Santos JE. Matrix metalloproteinases as drug targets in preeclampsia. Curr Drug Targets 2013; 14: 325-334.

37 Reference Values for Arterial Stiffness' Collaboration. Determinants of pulse wave velocity in healthy people and in the presence of cardiovascular risk factors: 'establishing normal and reference values'. Eur Heart J 2010; 31: 2338-2350.

38 Cecelja M, Chowienczyk P. Dissociation of aortic pulse wave velocity with risk factors for cardiovascular disease other than hypertension: a systematic review. Hypertension 2009; 54: 1328-1336.

39 Vlachopoulos C, Xaplanteris P, Vyssoulis G, Bratsas A, Baou K, Tzamou V, Aznaouridis K, Dima I, Lazaros G. Stefanadis C. Association of serum uric acid level with aortic stiffness and arterial wave reflections in newly diagnosed, never-treated hypertension. Am J Hypertens 2010; 24: 33-93.

40 Bian S, Guo H, Ye P, Luo L, Wu H, Xiao W. Serum uric Acid level and diverse impacts on regional arterial stiffness and wave reflection. Iran J Public Health 2012; 41: 33-41.

41 Kanellis J, Watanabe S, Li JH, Kang DH, Li P, Nakagawa T, Wamsley A, Sheikh-Hamad D, Lan HY, Feng L, Johnson RJ. Uric acid stimulates monocyte chemoattractant protein-1 production in vascular smooth muscle cells via mitogen-activated protein kinase and cyclooxygenase-2. Hypertension 2003; 41: 1287-1293.

42 Watanabe K, Mori T, Iwasaki A, Kimura C, Matsushita H, Shinohara K, Wakatsuki A. Increased oxidant generation in the metabolism of hypoxanthine to uric acid and endothelial dysfunction in early-onset and late-onset preeclamptic women. Matern Fetal Neonatal Med 2012; 25: 2662-2666.

43 Powe CE, Levine RJ, Karumanchi SA. Preeclampsia, a disease of the maternal endothelium: the role of anti-angiogenic factors and implications for later cardiovascular disease. Circulation 2011; 123: 2856-2869.

44 Sonoda H, Takase H, Dohi Y, Kimura G. Factors associated with brachial-ankle pulse wave velocity in the general population. J Hum Hypertens 2012; 26: 701-705.

45 McIntyre NJ, Fluck RJ, McIntyre CW, Fakis A, Taal MW. Determinants of arterial stiffness in chronic kidney disease stage 3. PLoS One 2013; 8: e55444.

46 Khalil AA, Cooper DJ, Harrington KF. Pulse wave analysis: a preliminary study of a novel technique for the prediction of pre-eclampsia. BJOG 2009; 116: 268-276.

47 Khalil A, Jauniaux E, Harrington K. Antihypertensive therapy and central hemodynamics in women with hypertensive disorders in pregnancy. Obstet Gynecol 2009; 113: 646-654.

48 Yinon Y, Kingdom JC, Odutayo A, Moineddin R, Drewlo S, Lai V, Cherney DZ, Hladunewich MA. Vascular dysfunction in women with a history of preeclampsia and intrauterine growth restriction: insights into future vascular risk. Circulation 2010; 122: $1846-1853$

49 Poon LC, Kametas NA, Chelemen T, Leal A, Nicolaides KH. Maternal risk factors for hypertensive disorders in pregnancy: a multivariate approach. J Hum Hypertens 2010; 24: 104-110.

50 Phillips JK, Janowiak M, Badger GJ, Bernstein IM. Evidence for distinct preterm and term phenotypes of preeclampsia. J Matern Fetal Neonatal Med 2010; 23: $622-626$

51 Xiong X, Demianczuk NN, Saunders LD, Wang FL, Fraser WD. Impact of preeclampsia and gestational hypertension on birth weight by gestational age. Am J Epidemiol 2002 155: 203-209.

52 Roberts JM, Catov JM. Preeclampsia more than 1 disease or is it? Hypertension 2008, 51: 989-990.

53 Kucukgoz Gulec U, Ozgunen FT, Buyukkurt S, Guzel AB, Urunsak IF, Demir SC, Evruke IC. Comparison of clinical and laboratory findings in early- and late-onset preeclampsia. J Matern Fetal Neonatal Med 2013; 26: 1228-1233.

54 Murphy DJ, Stirrat GM. Mortality and morbidity associated with early-onset preeclampsia. Hypertens Pregnancy 2000; 19: 221-231.

55 Carty DM, Neisius U, Rooney LK, Dominiczak AF, Delles C. Pulse wave analysis for the prediction of preeclampsia. J Hum Hypertens 2014; 28: 98-104.

56 Robb AO, Mills NL, Din JN, Smith IB, Paterson F, Newby DE, Denison FC. Influence of the menstrual cycle, pregnancy, and preeclampsia on arterial stiffness. Hypertension 2009; 53: 952-958.

57 Rana S, Karumanchi SA, Levine RJ, Venkatesha S, Rauh-Hain JA, Tamez H, Thadhani R. Sequential changes in antiangiogenic factors in early pregnancy and risk of developing preeclampsia. Hypertension 2007; 50: 137-142.

58 Moore Simas TA, Crawford SL, Solitro MJ, Frost SC, Meyer BA, Maynard SE. Angiogenic factors for the prediction of preeclampsia in high-risk women. Am J Obstet Gynecol 2007; 197: 244.e1-244.e8. 